\section{$\alpha$-Lipoic acid treatment prevents cystine urolithiasis in a mouse model of cystinuria}

\author{
Tiffany Zee ${ }^{1,2}$, Neelanjan Bose ${ }^{1,2}$, Jarcy Zee ${ }^{3}$, Jennifer N Beck ${ }^{1,2}$, \\ See Yang ${ }^{2}$, Jaspreet Pariharr, Min Yang ${ }^{5}$, Sruthi Damodar ${ }^{2}$, \\ David Hall ${ }^{2}$, Monique N O'Leary², Arvind Ramanathan', \\ Roy R Gerona ${ }^{6}$, David W Killilea ${ }^{1,7}$, Thomas $\mathrm{Chi}^{1}{ }^{1}$, Jay Tischfield ${ }^{5}$, \\ Amrik Sahota $^{5}$, Arnold Kahn ${ }^{2}$, Marshall L Stoller ${ }^{1,8}$ \& \\ Pankaj Kapahi ${ }^{1,2,8}$
}

\section{Cystinuria is an incompletely dominant disorder characterized by defective urinary cystine reabsorption that results in the formation of cystine-based urinary stones. Current treatment options are limited in their effectiveness at preventing stone recurrence and are often poorly tolerated. We report that the nutritional supplement $\alpha$-lipoic acid inhibits cystine stone formation in the SIc $3 a 1^{-/-}$mouse model of cystinuria by increasing the solubility of urinary cystine. These findings identify a novel therapeutic strategy for the clinical treatment of cystinuria.}

Kidney stones affect approximately $9 \%$ of the US population, with rates of incidence and prevalence increasing within the United States and globally ${ }^{1}$. While the surgical techniques used to remove obstructive stones have improved, few therapeutic advances have been made to prevent stone recurrence ${ }^{2}$. Cystinuria is a rare type of kidney stone disease caused by mutations in the SLC3A1 and/or SLC7A9 genes, which are responsible for cystine reabsorption in the renal proximal tubule, and is characterized by aggressive and recurrent cystine stone formation. Currently available interventions aimed at preventing the formation of cystine stones in these individuals include increasing fluid intake and treatment with pharmaceutical compounds that increase urinary $\mathrm{pH}$ or interfere with cysteine dimerization ${ }^{3}$. However, these measures have marginal effects on stone prevention, and the medications are poorly tolerated with adverse side effects that may be serious ${ }^{4,5}$. Furthermore, cystine stones are dense, large, and mostly resistant to extracorporeal shockwave lithotripsy (SWL), with obstructive stones often requiring multiple procedures or surgery for removal $^{6}$. Thus, the high rate of stone recurrence and the surgical burden associated with cystinuria place these patients at an increased risk for reduced kidney function and chronic kidney damage ${ }^{7}$.

To address the clinical challenges associated with cystinuria, we used the Slc3a1-1- mouse model, which develops cystine urolithiasis, to identify compounds that effectively inhibit stone formation ${ }^{8}$.
Micro-computed tomography $(\mu \mathrm{CT})$ analysis revealed that $S l c 3 a 1^{-/-}$ mice accumulated urinary bladder stones at an average rate of $1 \mathrm{~mm}^{3} / \mathrm{d}$ (Fig. 1a). We applied this measure to evaluate compounds for their effects on cystine stone growth in vivo. Notably, mice treated with the drug tiopronin, a sulfhydryl drug approved for treatment of cystinuria, showed no significant difference in cystine stone growth rate when compared to mice on a regular diet (Supplementary Fig. 1a). This result is consistent with observations that tiopronin has a limited therapeutic effect ${ }^{9}$. Similarly, treatment of mice with L-cystine dimethylester (L-CDME), an inhibitor of cystine crystal growth that has previously been shown to result in smaller stone size distrubtion $^{8,10}$, also had no significant effect on cystine stone growth rate when compared to a regular diet (Supplementary Fig. 1b). To identify more effective inhibitors, we evaluated compounds to inhibit cystine stone formation on the basis of two criteria: (i) activation of the antioxidant response signaling pathway that promotes glutathione synthesis and cellular cystine uptake and (ii) successful in vivo delivery of the compound to the renal proximal tubules and urine. Treatment with the pro-antioxidant sulforaphane ${ }^{11}$ had a modest effect on cystine stone growth (Supplementary Fig. 1c). In contrast, we found that the pro-antioxidant compound $\alpha$-lipoic acid ( $\alpha$-LA) was a strong suppressor of stone growth, as mice treated with $\alpha$-LA had lower growth rates for stone formation than untreated mice (Fig. 1b).

We treated 4- to 7-week-old male Slc3a1 ${ }^{-/}$mice, before any stone formation, with oral administration of $\alpha$-LA $(0.5 \%)$ as a supplement to their diet. While all untreated Slc3a1 ${ }^{-/-}$mice developed stones within 6 weeks of the start of the study and continued to progressively accrue stone volume, mice treated with $\alpha$-LA had significantly delayed stone formation, exhibited lower overall stone volume accumulation, and formed fewer stones (Fig. 1c and Supplementary Fig. 1d,e). Further, $\alpha$-LA was an effective suppressor of cystine stone growth, as the growth rate of existing stones in $S l c 3 a 1^{-/-}$mice was lower when the mice were treated with $\alpha$-LA. Withdrawal of $\alpha$-LA over a period of 4 weeks resulted in reversion of stone growth rate to the initial rate of growth (Fig. 1d), suggesting that continuous $\alpha$-LA treatment is necessary for its effect on stone inhibition.

Mouse intake of a diet supplemented with $0.5 \% \alpha$-LA is approximately equivalent to a human dose of $2,700 \mathrm{mg} / \mathrm{d}$ for a $67-\mathrm{kg}$ adult ${ }^{12}$. While no human toxicity limit for $\alpha$-LA supplementation has been established, we tested whether lower doses of $\alpha$-LA effectively inhibited stone growth in the Slc3a1 $1^{-1-}$ mouse. $0.1 \%$ and $0.25 \% \alpha$-LA in the mouse diet, approximately equivalent to human doses of 540 and $1,350 \mathrm{mg} / \mathrm{d}$, respectively ${ }^{12}$, significantly attenuated cystine stone growth when compared to no treatment (Fig. 1e). However, these moderate doses were also significantly less effective than the high dose of $0.5 \% \alpha$-LA in the mouse diet, as they caused slower growth,

\footnotetext{
${ }^{1}$ Department of Urology, University of California, San Francisco, San Francisco, California, USA. ${ }^{2}$ Buck Institute for Research on Aging, Novato, California, USA. ${ }^{3}$ Arbor Research Collaborative for Health, Ann Arbor, Michigan, USA. ${ }^{4}$ Division of Urology, Robert Wood Johnson Medical School, Rutgers University, New Brunswick, New Jersey, USA. ${ }^{5}$ Department of Genetics and the Human Genetics Institute of New Jersey, Rutgers University, Piscataway, New Jersey, USA. ${ }^{6}$ Department of Obstetrics, Gynecology, and Reproductive Sciences, University of California, San Francisco, San Francisco, California, USA. ${ }^{7}$ Nutrition and Metabolism Center, Children's Hospital of Oakland Research Institute, Oakland, California, USA. ${ }^{8}$ These authors contributed equally to this work. Correspondence should be addressed to M.L.S. (marshall.stoller@ucsf.edu) or P.K. (pkapahi@buckinstitute.org).
}

Received 27 June 2016; accepted 9 January 2017; published online 6 February 2017; doi:10.1038/nm.4280 
a

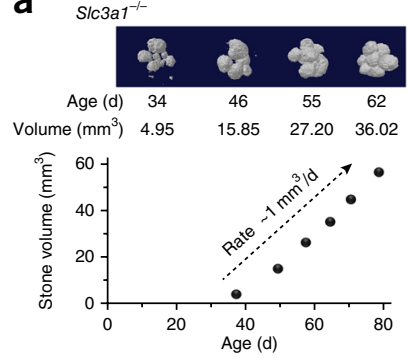

C

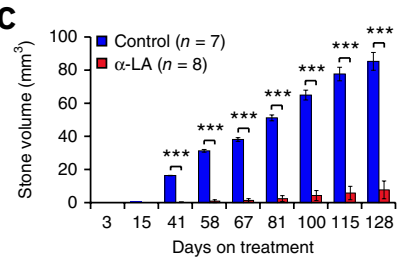

d

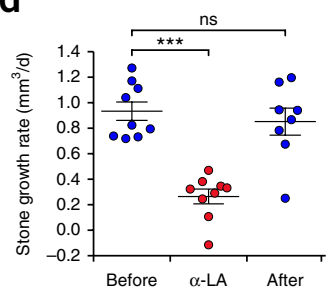

b
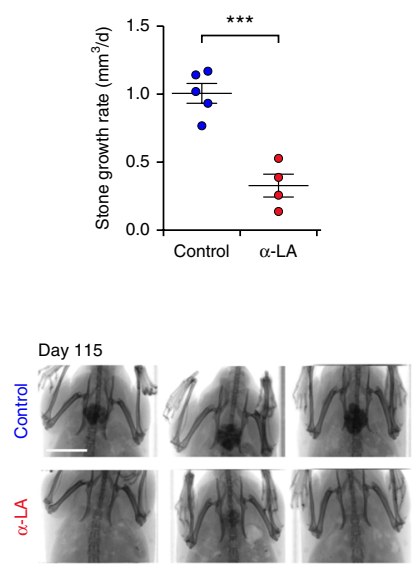

e

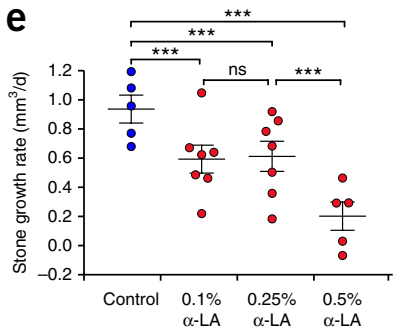

Figure $1 \alpha$-Lipoic acid inhibits cystine stone formation in the S/c3a1 ${ }^{-/-}$ mouse model of cystinuria. (a) In vivo bladder stone growth in a representative $(n=24) S / c 3 a 1^{-1-}$ male mouse, quantified by $\mu \mathrm{CT}$ analysis. (b) Stone growth rate in stone-forming S/c3a $1^{-1-}$ mice treated with $\alpha$-LA $(0.5 \% \mathrm{w} / \mathrm{w}$ supplementation in the diet) $(n=4)$ as compared to control, untreated mice $(n=5)$. Statistical differences in stone growth rate relative to the control were tested using a linear mixed regression model. (c) Left, stone volume in $S / \mathrm{c} \mathrm{al}^{-/-}$mice reared on a diet supplemented with $\alpha$-LA $(n=8)$ as compared to untreated control mice

$(n=7)$. Statistical differences in stone volume between $\alpha$-LA-treated and control mice at each time point were determined by Student's t-test after adjusting for multiple comparisons (Online Methods).

Right, representative radiograph images ( $n=361 \mu \mathrm{CT}$ scans) of the pelvic region of control mice and $\alpha$-LA-treated mice on day 115 of treatment. Results are representative of three independent experiments. Scale bar, $1 \mathrm{~cm}$. (d) Stone growth rate in pretreatment, $\alpha$-LA treatment, and post-treatment periods as evaluated by weekly $\mu$ CT scan for 6 weeks in S/c3a1-/- mice $(n=9)$. Statistical differences in stone growth rate between treatment periods were tested using a linear mixed regression model with linear spline. (e) Stone growth rate in S/c3a $1^{-/-}$mice upon administration of different doses of $\alpha-\mathrm{LA}$ (control, $n=5 ; 0.1 \% \alpha-\mathrm{LA}$, $n=7 ; 0.25 \% \alpha$-LA, $n=7 ; 0.5 \% \alpha-\mathrm{LA}, n=5$ ). Statistical differences in stone growth rate were tested using a linear mixed regression model. Data are presented as means \pm s.e.m. and are plotted for individual mice. $* * * P<0.001 . \mathrm{ns}$, not significant.

indicating that the effect of $\alpha$-LA on cystine stone growth was dose dependent (Fig. 1e). Of note, clinical trials assessing daily treatment with $\alpha$-LA at doses of $600 \mathrm{mg}$ to $1,800 \mathrm{mg}$ have reported no major adverse reactions ${ }^{13}$.

$\alpha$-LA treatment protected the kidneys of $S l c 3 a 1^{-/-}$mice from atrophy and hydronephrosis-related damage ${ }^{14}$ (Supplementary Fig. 2), likely by preventing the formation of obstructive stones. Slc $3 a 1^{-/-}$ mice treated with $\alpha$-LA had normal body weight and modestly greater food and water intake when compared to mice that were not treated with $\alpha$-LA (Supplementary Fig. 3a-c), suggesting that $\alpha$ LA does not prevent the formation of stones through an anorexic

mechanism ${ }^{15}$ or by promoting hydration. Additionally, urinary $\mathrm{pH}$ was not significantly different in mice treated with $\alpha$-LA when compared to untreated controls (Supplementary Fig. 3d).

$\alpha$-LA treatment did not affect urinary cystine concentration in Slc3a1 $1^{-1-}$ mice, as cystine levels in control versus $\alpha$-LA-treated mice were not significantly different (Fig. 2a). This result suggests that $\alpha$-LA (or its reduced form, dihydrolipoic acid) does not chelate cysteine or interfere with cysteine dimerization, as is the mechanism of thiol-binding drugs like tiopronin and penicillamine.

We then tested the hypothesis that $\alpha$-LA inhibits cystine stone formation by promoting cystine transport and metabolism. $\alpha$-LA treatment has been shown to increase Nrf2 nuclear localization and the transcription of Nrf2-regulated genes that promote glutathione biosynthesis via cystine import and cellular cysteine utilization ${ }^{16,17}$. We examined whether the effects of $\alpha$-LA on cystine stone growth

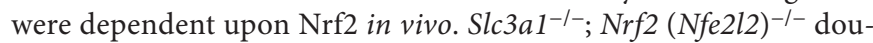
ble-knockout mice were treated with $\alpha$-LA and stone growth in these mice was compared to that in $S l c 3 a 1^{-1-}$ control littermates. Nrf2 did not contribute to cystine stone formation, as the rate of stone growth in $\mathrm{Slc}_{\mathrm{a} a 1^{-/-}}$Nrf2 $\mathrm{N}^{-/-}$mice was not significantly different from that in $S l c 3 a 1^{-/-}$mice (Fig. 2b). Additionally, the effect of $\alpha$-LA on stone growth was maintained in $\mathrm{Slc3a1^{-/- }}$; $\mathrm{rff}^{-/-}$mice, indicating that $\alpha$-LA regulates cystine stone formation independently of its role in activating the Nrf2-dependent antioxidant response (Fig. 2b).

On the basis of these results and the clinical observations that $\alpha$-LA administration alters urine attributes ${ }^{18}$, we examined whether $\alpha$-LA affected cystine solubility in the urine. Ex vivo cystine precipitation analysis revealed that cystine was considerably more soluble in the urine of $\alpha$-LA-treated mice than it was in the urine of untreated mice (Fig. 2c). However, while previous studies have shown that $\mathrm{L}$-CDME interferes with cystine crystal growth and results in crystals of a distinctly different shape ${ }^{10}$, treatment with $\alpha$-LA did not alter the cystine hexagonal crystal habit (Supplementary Fig. 4). Furthermore, synthetic $\alpha$-LA had no detectable effect on cystine solubility, suggesting that $\alpha$-LA does not directly interfere with cystine precipitation (Fig. 2d). This result is consistent with the observation that exogenous $\alpha$-LA is known to undergo extensive metabolism in both mice and humans, including $\beta$-oxidation, sulfur methylation, sulfur oxidation, and glycine conjugation in mice, before being excreted into the urine ${ }^{19}$. It is likely that these $\alpha$-LA-derived metabolites in the urine are ultimately responsible for preventing cystine stone formation in $S l c 3 a 1^{-/-}$mice. Further investigation will be needed to identify the specific metabolite(s) of $\alpha$-LA responsible for increasing cystine solubility and to determine how these $\alpha$-LA-induced changes affect cystine crystallization.

We report here a new therapeutic application of $\alpha$-LA treatment that protects against cystine stone formation in a mouse model of cystinuria. While $\alpha$-LA has previously been implicated as a potential therapeutic for its beneficial effects in promoting stress-activated signaling mechanisms, our data identify a novel function of $\alpha$-LA that relies upon increasing urinary cystine solubility, likely via the excretion of downstream $\alpha$-LA metabolites into the urine. This increase in cystine solubility is achieved without inducing changes to urinary $\mathrm{pH}$, which predispose to other types of stone formation (for example, calcium phosphate $)^{20}$. The fact that $\alpha$-LA is a widely available nutritional supplement with few adverse side effects ${ }^{13}$ makes its use potentially advantageous in comparison to that of currently available medications and renders it a particularly attractive candidate for assessment in individuals with cystinuria. These findings highlight a new avenue for treating and preventing cystine stone recurrence and have implications for understanding treatment approaches for other types of urinary stone disease. 


\section{BRIEF COMMUNICATIONS}
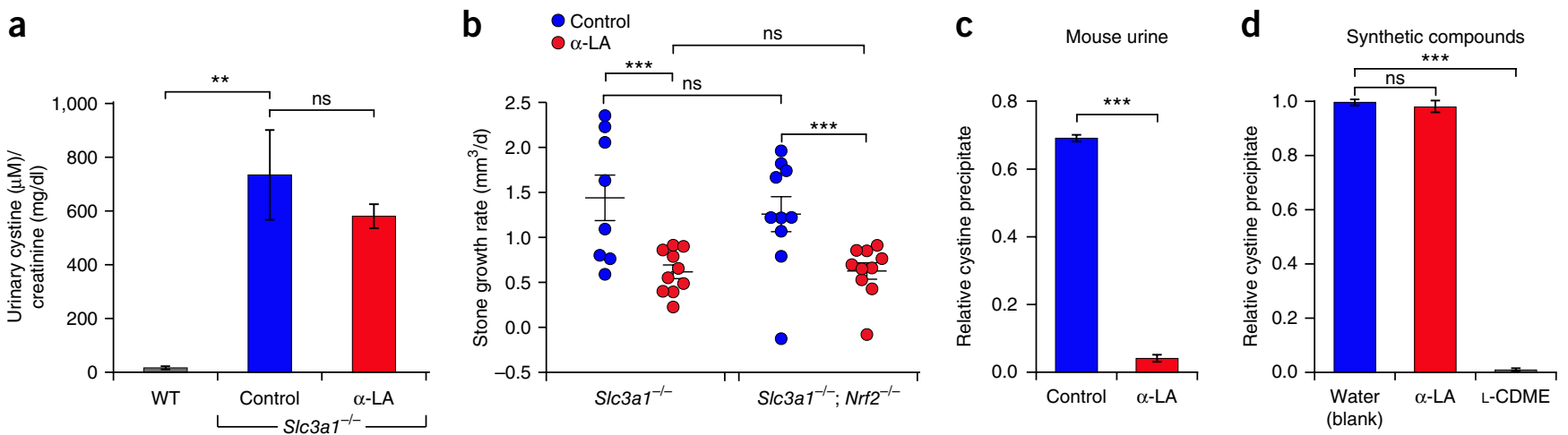

Figure $2 \alpha$-Lipoic acid increases cystine solubility in the urine environment. (a) Urinary cystine concentration for wild-type (WT) ( $n=2$ ), control S/c3a1-I- $(n=4)$, and $\alpha$-LA-treated S/c3a1 ${ }^{-I-}(n=5)$ mice. Values are normalized to creatinine measurements. Data are presented as means \pm s.e.m. Statistical differences in urinary cystine concentration were determined by Student's $t$-test. (b) Stone growth rates with $\alpha$-LA treatment in S/c3a ${ }^{-l-}$ mice (control, $n=8$; treated, $n=10$ ) and S/c3a1 ${ }^{-l-}$; Nrf2 $2^{-1-}$ mice (control, $n=10$; treated, $n=10$ ). Data are plotted for individual mice and are presented as means \pm s.e.m. Statistical differences in growth rate between $\alpha$-LA-treated mice and controls and the effect of $\alpha$-LA between S/c3a1 ${ }^{-/-}$and S/c3a $1^{-/-}$; Nrf2-Imice were tested using a linear mixed regression model. (c) Relative yield of L-cystine precipitate obtained after crystallization for $3 \mathrm{~d}$ in the presence of urine from untreated and $\alpha$-LA-treated wild-type mice as compared to a blank (water). Statistical differences were determined by Student's $t$-test. Data are presented as means \pm s.d. from three measurements. (d) Relative yield of L-cystine precipitate obtained after crystallization for $3 \mathrm{~d}$ in the presence of $\alpha$-LA $(500 \mu \mathrm{M})$ or L-cystine dimethyl ester ( $L-C D M E ; 500 \mu \mathrm{M})$, a positive control ${ }^{10}$, as compared to a blank (water). Statistical differences were determined by Student's $t$-test. Data are presented as means \pm s.d. from three measurements. ${ }^{* *} P<0.01,{ }^{* * *} P<0.001$. ns, not significant.

\section{METHODS}

Methods, including statements of data availability and any associated accession codes and references, are available in the online version of the paper.

Note: Any Supplementary Information and Source Data files are available in the online version of the paper.

\section{ACKNOWLEDGMENTS}

We thank R. Murphy and S. Melov for their advice regarding $\mu \mathrm{CT}$; T. Te Koi for histology assistance; M. Chamoli and N. Mathew for help with imaging; and D. Chrzan for helpful discussions. This work was supported by grants from the American Federation of Aging Research (to P.K.), the Larry L. Hillblom Foundation (to P.K.), the Boston Scientific Foundation (to M.L.S.), and the NIH (R01 AG038688 and R01 AG045835 to P.K.; R21 DK091727 to P.K. and M.L.S.; P20 DK100863 and R21 DE025961 to M.L.S.; K12 DK083021 and R21 DK109433 to T.C.).

\section{AUTHOR CONTRIBUTIONS}

T.Z., A.K., M.L.S., and P.K. conceived the experiments. T.Z. and N.B. developed methodology and designed experiments. T.Z., N.B., and J.Z. analyzed and interpreted data. T.Z., J.N.B., S.Y., J.P., M.Y., and S.D. performed in vivo experiments. N.B., T.Z., D.H., and A.R. performed ex vivo and in vitro experiments. M.N.O'L. and T.Z. performed food and water intake measurements. J.T. and A.S. designed and generated the $\mathrm{Slc}_{\mathrm{Ca1}} \mathrm{1}^{-/-}$mouse. T.Z., N.B., and J.Z. wrote the manuscript, and D.W.K., A.S., R.R.G., T.C., A.K., M.L.S., and P.K. revised the manuscript.

\section{COMPETING FINANCIAL INTERESTS}

The authors declare no competing financial interests.

Reprints and permissions information is available online at http://www.nature.com/ reprints/index.html.

1. Scales, C.D. Jr., Smith, A.C., Hanley, J.M. \& Saigal, C.S. Eur. Urol. 62, 160-165 (2012).

2. Morgan, M.S. \& Pearle, M.S. Br. Med. J. 352, i52 (2016).

3. Mattoo, A. \& Goldfarb, D.S. Semin. Nephrol. 28, 181-191 (2008).

4. Zheng, Z. et al. Ren. Fail. 36, 1455-1460 (2014).

5. Ishak, R. \& Abbas, O. Am. J. Clin. Dermatol. 14, 223-233 (2013).

6. Varda, B.K. et al. J. Pediatr. Urol. 12, 106 (2016).

7. Rule, A.D., Krambeck, A.E. \& Lieske, J.C. Clin. J. Am. Soc. Nephrol. 6, 2069-2075 (2011).

8. Sahota, A. et al. Urology 84, 1249 (2014).

9. Becker, G., Caring for Australians with Renal Impairment (CARI)lpar Nephrology (Carlton) 12 (Suppl. 1), S4-S10 (2007).

10. Rimer, J.D. et al. Science 330, 337-341 (2010).

11. Yoon, H.Y. et al. Biochem. Pharmacol. 75, 2214-2223 (2008).

12. Reagan-Shaw, S., Nihal, M. \& Ahmad, N. FASEB J. 22, 659-661 (2008).

13. Ziegler, D. et al. Diabetes Care 29, 2365-2370 (2006)

14. Livrozet, M. et al. PLoS One 9, e102700 (2014).

15. Kim, M.S. et al. Nat. Med. 10, 727-733 (2004).

16. Suh, J.H. et al. Proc. Natl. Acad. Sci. USA 101, 3381-3386 (2004).

17. Han, D. et al. Biofactors 6, 321-338 (1997).

18. Teichert, J. et al. J. Clin. Pharmacol. 45, 313-328 (2005).

19. Schupke, H. et al. Drug Metab. Dispos. 29, 855-862 (2001).

20. Wagner, C.A. \& Mohebbi, N. J. Nephrol. 23 (Suppl. 16), S165-S169 (2010). 


\section{ONLINE METHODS}

Animals. Homozygous Slc3a1 ${ }^{-1-}$ male mice (on a mixed C57Bl/6 and 129/Sv) background) were bred and maintained as previously described ${ }^{21}$. Specifically, Slc3a1 $1^{-1-}$ mice were generated by homologous recombination in embryonic stem (ES) cells. A $24-\mathrm{kb}$ DNA fragment was isolated by screening a $129 / \mathrm{SvJ}$ genomic library. A targeting vector was constructed by replacing $5.7 \mathrm{~kb}$ of the wild-type sequence that included exon 1 with a $1.7-\mathrm{kb}$ neomycin cassette under the control of a PGK1 promoter. The vector also included a 2.9-kb $5^{\prime}$ homology arm, a 5.6-kb 3' homology arm, and a thymidine kinase sequence upstream of the $5^{\prime}$ homology arm. Southern blotting with $5^{\prime}$ and $3^{\prime}$ screening probes located external to the homology arms was used to verify the difference in fragment size between the wild-type (24-kb) and knockout (20-kb) alleles. The targeting vector was linearized with the restriction enzyme AflII and then electroporated into 129/SvJ ES cells. Recombinant clones were identified using the $5^{\prime}$ and $3^{\prime}$ probes, and the presence of the PGK-neo cassette in the knockout allele was confirmed using a neo probe. For each experiment, Slc $3 a 1^{-1-}$ littermate mice were randomly assigned to treatment or control groups. Wild-type (129X1/SvJ) and $\mathrm{Nrf2}^{-/-}$mice were purchased from Jackson Laboratories, and

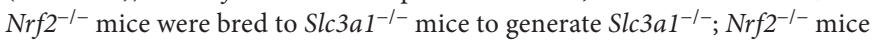
and Slc3a1 ${ }^{-1-} ; \mathrm{Nrf2}^{+/+}$littermate controls.

$\alpha$-LA was administered via $0.5 \%, 0.25 \%$, or $0.1 \%(\mathrm{w} / \mathrm{w})$ supplementation in the diet (Envigo); control mice were administered a nonsupplemented equivalent diet. Tiopronin, $\mathrm{N}$-(2-mercaptopropionyl)glycine, was administered via daily oral gavage at $3.7 \mathrm{mg} / \mathrm{d}$, the mouse dose equivalent to the tiopronin dose of $1,000 \mathrm{mg} / \mathrm{d}$ prescribed to patients, calculated according to the body surface area normalization method ${ }^{12}$. L-CDME was administered via daily oral gavage at $2 \mathrm{mg} / \mathrm{d}$, as was previously done ${ }^{8}$. Control mice for these experiments were administered water only as a vehicle control. Sulforaphane was administered via intraperitoneal injection three times per week at $25 \mathrm{mg} / \mathrm{kg}$ in PBS. Control mice were administered PBS only as a vehicle control.

Food and water intake over $24 \mathrm{~h}$ were measured using group-housed mice (3- 4 mice per cage) in metabolic cages (Promethion). For urinary pH determination, urine from Slc3a1 ${ }^{-1-}$ mice was collected from voluntary expulsion and measured using a micro combination $\mathrm{pH}$ electrode (Lazar).

All experiments detailed here involving the use of animals were reviewed and approved by the Institutional Animal Care and Use Committees (IACUC) of the Buck Institute and Rutgers University.

Micro-computed tomography. Stone growth rate was calculated from weekly measurements taken during a period of 6 weeks. As Slc3a1 $1^{-1-}$ mice are susceptible to mortality, measurements from time points immediately before any deaths were excluded from analysis ${ }^{14,22}$. Mice were anesthetized and scanned weekly using the Skyscan 1176 microcomputed tomography $(\mu \mathrm{CT})$ scanner (Bruker). The Skyscan reconstruction program NRecon was used for image reconstruction, and bladder stone volume was quantified using the Bruker CT-Analyzer (CTAn, Version 1.14) program. 3D image models were created using CTAn and Bruker CT-Volume (CTVol, Version 2.2).

Kidney histology. Kidney tissues were fixed in formalin and embedded in paraffin. $7-\mu \mathrm{m}$ sections were then stained with $\mathrm{H} \& \mathrm{E}$.

Cystine determination. Urine from $\mathrm{Slc} 3 a 1^{-/-}$mice was collected from voluntary expulsion, and internal standard DL-cystine (3,3,3',3'-D4, Cambridge Isotope Laboratories) was added immediately to the samples. Urine samples were then stored at $-80^{\circ} \mathrm{C}$ before cystine estimation. Urinary cystine was measured using the EZ-FAAST kit from Phenomenex coupled with liquid chromatography and tandem mass spectrometry (LC-MS/MS), following vendor-provided directions. Creatinine levels were determined using an assay kit according to the manufacturer's instructions (Cayman Chemical).

Cystine precipitation assays. $100 \mathrm{ml}$ of $4 \mathrm{mM}$ supersaturated L-cystine solution was prepared by heating the cystine suspension in water under reflux at $100^{\circ} \mathrm{C}$ for $20 \mathrm{~min}$ with continuous stirring to completely dissolve the $\mathrm{L}$-cystine, as previously described ${ }^{10} .400 \mu$ of this supersaturated L-cystine solution was added to borosilicate glass tubes ( 3 technical replicates per condition per experiment) containing $100 \mu$ l of water (blank), synthetic compounds (at least 3 independent experiments performed), or mouse urine (at least 2 independent experiments performed), and the tubes were vortexed briefly to homogenize the contents. The solutions were subsequently incubated at $4{ }^{\circ} \mathrm{C}$ for $3 \mathrm{~d}$ to allow cystine precipitation. At the end of the incubation period, the tubes were centrifuged at 4,000 r.p.m. for $20 \mathrm{~min}$ and the supernatant was discarded. The remaining cystine precipitate was dissolved in $3.6 \mathrm{ml}$ of $25 \mu \mathrm{M}$ D4-cystine (internal standard) solution, and $2 \mu \mathrm{l}$ was injected for LC-MS/MS analysis. Mouse urine samples were obtained from 24-h urine collections from 129X1/SvJ male mice treated with or without $0.5 \% \alpha$-LA (Tecniplast).

Liquid chromatography and tandem mass spectrometry. LC was performed using a Shimadzu UFLC prominence system fitted with modules CBM-20A (Communication bus module), DGU- $\mathrm{A}_{3}$ (degasser), two LC-20AD (liquid chromatograph, binary pump), and SIL-20AC HT (auto sampler) and connected to a Phenomenex Luna $\mathrm{NH}_{2}$ column $(2 \times 150 \mathrm{~mm}, 3 \mu \mathrm{m}, 100 \AA)$. MS was performed using a 4000 QTRAP LC-MS/MS mass spectrometer from AB SCIEX fitted with a Turbo V ion source. AB Sciex's Analyst v1.6.1 was used for all forms of data acquisition, development of the LC method, and optimization of analyte-specific MRM (multiple-reaction monitoring) transitions. AB Sciex's Peakview v2.1 and Skyline v3.5 (ref. 23) were used for LC-MS/MS data analysis.

Optimization of cystine- and D4-cystine-specific MRM transitions, such as determination of suitable precursor and product ions and optimal MS parameters for each transition $\left(Q_{1}\right.$, precursor $\rightarrow Q_{3}$, product), was achieved by isocratic flow injection of the $10 \mu \mathrm{M}$ solution for each compound. The most intense $\left(Q_{1} \rightarrow Q_{3}\right)$ transition was used as the quantifier whereas the next best transition was used as the qualifier for each compound (Supplementary Table 1). For LC separation, a solvent gradient of $95 \% 20 \mathrm{mM}$ ammonium acetate $/ 20 \mathrm{mM}$ ammonium hydroxide ( $\mathrm{pH} \sim 9.5$ ) in water and $5 \%$ acetonitrile (aqueous)-acetonitrile (organic) was used with a $0.4 \mathrm{ml} / \mathrm{min}$ flow rate, starting with an acetonitrile content of $50 \%$ for $1.5 \mathrm{~min}$, which was decreased to $2 \%$ over $3.5 \mathrm{~min}$ and held at $2 \%$ for $1.5 \mathrm{~min}$. The LC column was subsequently reconstituted to its initial condition (organic content of 50\%) over the next $1 \mathrm{~min}$ and re-equilibrated for $4 \mathrm{~min}$. For cystine estimation (retention time $=4.9 \mathrm{~min}$ ), the mass spectrometer was operated in positive ion mode. Source conditions were as follows: curtain gas (CUR), 20; nebulizer gas (GS1), 60; auxiliary gas (GS2), 50; ionspray voltage (IS), 4,500 V; source temperature (TEM), $450{ }^{\circ} \mathrm{C}$. Quantification of cystine was based on integration of corresponding LC-MS/MS-specific quantifier peaks (peak areas) and the corresponding quantifier peak area for D4-cystine.

Statistical analyses. Littermate mice were randomly assigned to treatment or control groups for each experiment. Investigators were not blinded to group allocation. Most experiments included a control-only period before randomization to ensure mice would survive long enough for study. Those that did not survive to randomization were removed from the study $(n=7$ mice). Means and standard errors were calculated in each treatment and control group. Two-sided Student's $t$-tests were used to compare differences in mean stone volume at single time points, urinary cystine levels, and relative cystine precipitates. Experiments involving multiple $t$-tests were adjusted for multiple comparisons using the Benjamini-Hochberg-Yekutieli procedure, which controls for false discovery rate among correlated tests ${ }^{24}$. Linear mixed regression models were used to assess the associations between treatment groups and stone growth over time. Sample size calculations were conducted assuming linear mixed regression models with six time points during each treatment period and stone growth of $1 \mathrm{~mm}^{3}$ per day in the control group. Assuming stone growth of $0.6 \mathrm{~mm}^{3}$ per day in the treatment group, at least $n=4$ mice were required in each group to have at least $80 \%$ power to detect differences across treatment and control groups. Assuming stone growth of $0.7 \mathrm{~mm}^{3}$ per day, $n=6$ mice were needed in each group.

Given the approximately linear increase in stone volume, time was used as a continuous variable in linear mixed regression models. An interaction term between time and treatment group was included to test for differences in mean stone growth across treatment groups. For experiments with more than two treatment groups, interaction terms were first jointly tested across all treatment groups. If the overall test indicated a significant interaction, differences for each pair of treatment groups were tested. Random intercepts for each mouse were included in regression models to account for the correlated, repeated stone 
volume measures within mice. For experiments in which mice were switched from one diet to another, linear splines were included in models with knots at the times of switch to allow for different stone growth rates while on each diet. For experiments in which mice were randomized to $\alpha$-LA treatment or control groups after a control-only period, mean stone growth rates were adjusted for baseline stone growth, which was defined as the mean stone growth rate in each mouse before randomization. A three-way interaction between mouse group, treatment group, and time was used in the regression model to test whether Nrf2 loss modified the effect of $\alpha$-LA. Two-sided tests were used in all models.

Stone volumes over time were plotted before modeling to ensure volume increased approximately linearly in each experiment and the continuous form of time in models was appropriate. Random intercepts and residuals estimated from models were plotted using histograms and quantile-quantile plots to check for normality. There was no evidence of any violations of these model assumptions. A Poisson regression model was used to compare the counts of stones between the control and $\alpha$-LA-treated groups. Model assumptions, including overdispersion and zero inflation, were checked and there was no evidence of assumption violations. All statistical analyses were conducted using R 3.2.3 software (R Foundation for Statistical Computing).

Data availability. Source data are available online for Figures $\mathbf{1}$ and $\mathbf{2 .}$

21. Ercolani, M. et al. Int. Urol. Nephrol. 42, 57-63 (2010).

22. Feliubadalo, L. et al. Nat. Genet. 23, 52-57 (1999).

23. MacLean, B. et al. Bioinformatics 26, 966-968 (2010).

24. Benjamini, Y. \& Yekutieli, D. Ann. Stat. 29, 1165-1188 (2001). 